\title{
Análise do equilíbrio das variáveis macroeconômicas do regime de metas de inflação sob a abordagem de sistemas de equações em diferença.
}

\author{
Érika Capelato, \\ Depto de Economia, FCLAR, UNESP \\ 14800-901, Araraquara, SP \\ E-mail: erika@ fclar.unesp.br \\ André Luiz Correa \\ Depto de Economia, FCLAR, UNESP \\ 14800-901, Araraquara, SP \\ E-mail: andrelc@fclar.unesp.br
}

\begin{abstract}
Resumo: O regime monetário de metas de inflação adotado pelo Brasil em 1999 após uma crise cambial tem o objetivo de fazer um anúncio prévio de uma meta numérica para a inflação buscando com isso coordenar a formação de expectativas inflacionárias dos agentes e a fixação de salários e preços. Muitos trabalhos empíricos relacionados à avaliação do regime de metas de inflação no Brasil ou adotando modelos e simulações matemáticas para estudar a compatibilidade entre o regime de metas de inflação e políticas econômicas de natureza heterodoxa, surgiram de diversas correntes de pensamento econômico. O presente trabalho apresenta um modelo matemático com o objetivo de analisar o equilíbrio das variáveis macroeconômicas (diferencial de juros, variação da taxa de câmbio, hiato do produto e inflação) no contexto do regime de metas de inflação sob a ótica de propriedades matemáticas do sistema de equações em diferenças para o período 1999-2013. A análise dos resultados possibilitou apontar a existência de convergência do modelo para um equilíbrio no qual, o diferencial de juros tem sinal negativo e a variação da taxa de câmbio, o hiato do produto e a inflação sinais positivos.
\end{abstract}

\section{Introdução}

De maneira simplificada, o regime de metas de inflação pode ser definido como uma estratégia de condução da política monetária baseada no estabelecimento de uma meta para a inflação, no início de determinado período, que é anunciada publicamente pelo governo e cujo cumprimento deve ser responsabilidade do Banco Central ${ }^{1}$. Este regime visa uma maior transparência na condução da política monetária, baseada na busca pelo aprimoramento dos canais de comunicação entre o Banco Central e os agentes, para que possa haver um monitoramento e uma avaliação do desempenho da autoridade monetária. Desta forma, essas metas podem coordenar a formação de expectativas inflacionárias dos agentes e a fixação de salários e preços, atuando assim como uma âncora nominal tanto para a inflação atual como para as expectativas futuras.

Vários autores de diversas correntes de pensamento econômico debruçaram-se sobre a questão da avaliação do regime de metas de inflação após sua implementação no Brasil. Inicialmente, privilegiaram-se aspectos técnicos do modelo, com destaque para os trabalhos de [3] e [8]. Transcorridos alguns anos, houve um aumento substancial no número de trabalhos empíricos relacionados à avaliação do regime de metas de inflação no Brasil, em grade parte adotando um ponto de vista de crítica ao referido regime, como por exemplo [10]. Outra vertente de trabalhos tem procurado avaliar, por meio de modelos e simulações matemáticas, a compatibilidade entre o

\footnotetext{
${ }^{1}$ Em [9] o autor apresenta uma discussão sobre os principais regimes monetários e contém as principais referências sobre o tema.
} 
regime de metas de inflação e políticas econômicas de natureza heterodoxa, por exemplo, [6], [7] e [11]. Os modelos presentes nestes trabalhos, todavia, não exploram explicitamente variáveis associadas à questão fiscal ou ao setor externo, como a taxa de câmbio.

O regime de metas de inflação foi implementado no Brasil em julho de 1999. Após a estabilização da moeda obtida a partir de meados de 1994 com o Plano Real, a manutenção do processo de estabilização da inflação, inicialmente apoiada em uma âncora cambial ${ }^{2}$, precisou ser repensada em um contexto de crises econômicas em países em desenvolvimento que ao longo dos anos 1990 adotaram políticas de maior liberalização das contas externas.

As autoridades monetárias brasileiras optaram por escolher um índice de preços cheio como meta para inflação, neste caso o IPCA (Índice de Preços ao Consumidor Amplo, que compreende famílias com renda de até 40 salários mínimos). Outros países que adotaram o regime de metas de inflação escolheram como parâmetro alguma medida do núcleo da inflação, de modo a isolar efeitos de choques temporários sobre a inflação. O chamado núcleo da inflação consiste em uma forma de calcular a inflação que exclui ou confere menor importância aos aumentos provocados por fatores temporários ou casuais na composição do índice de preços. Essa técnica de cálculo é utilizada para que se tenha o real comportamento da inflação, considerando-se as variações que sejam consistentes com o comportamento de toda a economia, e não apenas fatores localizados.

A operação do regime de metas de inflação baseia-se em uma função de reação do Banco Central em respostas ao comportamento de variáveis macroeconômicas, como a tendência da inflação em relação à meta, o nível de produto e o setor externo. O instrumento de política monetária escolhido para atingir os objetivos das autoridades monetárias é a taxa de juros SELIC.

Neste trabalho propomos um modelo matemático com variáveis macroeconômicas com o objetivo de analisar se há um equilíbrio para o modelo, utilizando-se parâmetros obtidos empiricamente por metodologia estatística apropriada.

\section{Metodologia}

\section{II.1. Sistema de Equações em Diferenças}

Para [1] podemos dizer que $X^{*}$ é um ponto de equilíbrio do sistema autônomo da forma

$$
X_{t}=A X_{t-1}
$$

se $A X^{*}=X^{*}$ ou $(A-I) X^{*}=0$. Assim, se $(A-I)$ é não singular temos $X^{*}=0$. Por outro lado, se $(A-I)$ é singular, existe uma família de pontos de equilíbrio.

Para [5] podemos dizer que um sistema é estável, se quando ligeiramente perturbado de seu estado de equilíbrio, todos os movimentos subseqüentes permanecer em uma pequena vizinhança do equilíbrio. Se, além de ser estável, cada movimento suficientemente perto do ponto de equilíbrio converge para este quando $t \rightarrow \infty$, então o equilíbrio é assintoticamente estável.

Em [1], pg.194, é demonstrado que as propriedades de estabilidade de um ponto de equilíbrio qualquer $X^{*} \neq 0$ são as mesmas que a do ponto de equilíbrio $X^{*}=0$. Assim, vamos assumir que $X^{*}=0$ é o único ponto de equilíbrio da equação (1), a qual possui como solução, considerando $X(0)=X_{0}$ um dado inicial, $X(t)=A^{t} X_{0}$. Se tivermos $\lim _{t \rightarrow \infty} X(t)=0$, então toda solução de (1) tende ao vetor nulo, isto é, ao equilíbrio.

Para checar este limite, ou seja, a estabilidade assintótica, usamos o seguinte resultado.

\footnotetext{
${ }^{2}$ Para maiores detalhes sobre o Plano real ver [4].
} 
Teorema 1: Assumindo que $A$ é uma matriz quadrada qualquer, então $\lim _{t \rightarrow \infty} A^{t}=0$ se, e somente se, $|\lambda|<1$ para todo autovalor $\lambda$ de A.

Demonstração: Corolário 3.24 em [1].

Se considerarmos o seguinte sistema autônomo não-homogêneo:

$$
X_{t}=A X_{t-1}+G
$$

sua solução, pela formula da Variação das Constante, é da forma:

$$
X(t)=A^{t} \cdot X_{0}+\sum_{r=0}^{t-1} A^{r} \cdot G
$$

Para estudar o comportamento da solução (3) quando $t \rightarrow \infty$ usamos a Expansão de Neumann dada pelo próximo resultado.

Teorema 2: Seja $A$ é uma matriz quadrada qualquer com $|\lambda|<1$ para todo autovalor $\lambda$ de A. Então $(I-A)$ é não-singular e $\lim _{t \rightarrow \infty} \sum_{r=0}^{t-1} A^{r}=\sum_{r=0}^{\infty} A^{r}=(I-A)^{-1}$.

Demonstração: [1] pg.170.

Logo, se são satisfeitas as condições da Expansão de Neumann para o sistema (2) temos, passando o limite em (3):

$$
\lim _{t \rightarrow \infty} X(t)=(I-A)^{-1} G
$$

ou seja, a solução é assintoticamente estável e converge para o equilíbrio $(I-A)^{-1} G$.

\section{II.2. Modelo Econômico}

Para descrevermos a dinâmica existente entre a diferencial da taxa de juros, variação da taxa cambial, a taxa de inflação e o hiato do produto, consideraremos o seguinte modelo matemático em tempo discreto:

$$
\left\{\begin{array}{l}
y_{t}=\alpha_{0}+\alpha_{1} i_{i-1}+\alpha_{2} e_{t-1}+\alpha_{3} \pi_{t-1}+\alpha_{4} y_{t-1} \\
i_{i}=\beta_{0}+\beta_{1} i_{t-1}+\beta_{2} e_{t-1}+\beta_{3} \pi_{t-1}+\beta_{4} y_{t-1} \\
e_{t}=\gamma_{0}+\gamma_{1} i_{t-1}+\gamma_{2} e_{t-1}+\gamma_{3} \pi_{t-1}+\gamma_{4} y_{t-1} \\
\pi_{t}=\delta_{0}+\delta_{1} i_{t-1}+\delta_{2} e_{t-1}+\delta_{3} \pi_{t-1}+\delta_{4} y_{t-1}
\end{array}\right.
$$

onde, $\pi_{t}$ e taxa de inflação correspondente a variação do IPCA em \% no período $t ; e_{t}$ é a variação da taxa cambial para venda em R \$US\$, disponibilizada pelo Banco Central do Brasil no período $t$ em relação ao período $t-1 ; i_{t}$ é uma medida do diferencial da taxa de juros entre o Brasil e o exterior, definido como: $i_{t}=r^{d}-r^{f}$, em que $r^{d}$ é a taxa Selic (divulgada pelo Banco Central do Brasil) e $r^{f}$ é a taxa de juros dos fundos federais (FED Funds), disponibilizada pelo Federal 
Reserve no período $t$ e $y_{t}$ é o hiato do produto obtido a partir da aplicação do filtro HodrickPrescott $^{3}$ aos dados do Produto Interno Bruto (PIB) calculados pelo Banco Central do Brasil no período $t$. Uma interpretação simples para $y_{t}$ neste trabalho, consiste em entendê-lo como o desvio do PIB de sua tendência de longo prazo.

O sistema de equações em diferença (4) pode ser reescrito da seguinte forma:

$$
X_{t}=A \cdot X_{t-1}+G
$$

sendo, $X_{t}=\left[\begin{array}{c}y_{t} \\ i_{t} \\ e_{t} \\ \pi_{t}\end{array}\right], A=\left[\begin{array}{cccc}\alpha_{1} & \alpha_{2} & \alpha_{3} & \alpha_{4} \\ \beta_{1} & \beta_{2} & \beta_{3} & \beta_{4} \\ \gamma_{1} & \gamma_{2} & \gamma_{3} & \gamma_{4} \\ \delta_{1} & \delta_{2} & \delta_{3} & \delta_{4}\end{array}\right], X_{t-1}=\left[\begin{array}{c}y_{t-1} \\ i_{t-1} \\ e_{t-1} \\ \pi_{t-1}\end{array}\right]$ e $G=\left[\begin{array}{c}\alpha_{0} \\ \beta_{0} \\ \gamma_{0} \\ \delta_{0}\end{array}\right]$.

A equação (5) descreve um sistema de equações em diferenças de ordem um não-homogêneo e autônomo, cuja solução é dada por:

$$
X\left(t, 0, X_{0}\right)=[A]^{t} \cdot X_{0}+\sum_{r=0}^{t-1}[A]^{r} \cdot G
$$

obtida pela formula da Variação das Constantes, Teorema 3.17 em [1], onde $X(0)=X_{0}$ é o dado inicial.

Nosso objetivo é analisar se há um equilíbrio intertemporal e estudar a estabilidade para o sistema (4), ou seja, tomar o limite quando $t \rightarrow \infty$ na equação (6) e verificar se haverá convergência para o equilíbrio ao longo do tempo.

Para obtermos os valores dos parâmetros, e assim, os coeficientes das matrizes $A$ e $G$, utilizamos o método econométrico descrito abaixo.

\section{II.2. Método Econométrico}

Os dados utilizados como parâmetros nas matrizes $A$ e $G$ foram obtidos por um processo de estimação que compreendeu o período de julho de 1999 a outubro de 2013, com periodicidade mensal totalizando 172 observações. Para obtermos as estimativas utilizamos o método das séries temporais multivariadas VAR (vetores auto regressivos) ${ }^{4}$ e através do software livre GRETL $^{5}$ obtivemos os seguintes parâmetros para o sistema (4).

$$
\left\{\begin{array}{l}
y_{t}=0,181-0,138 i_{i-1}+0,194 e_{t-1}-0,056 \pi_{t-1}+0,527 y_{t-1} \\
i_{i}=0,050+0,923 i_{t-1}+0,025 e_{t-1}+0,057 \pi_{t-1}-0,009 y_{t-1} \\
e_{t}=0,012-0,0008 i_{t-1}-0,050 e_{t-1}-0,017 \pi_{t-1}+0,007 y_{t-1} \\
\pi_{t}=0,125+0,063 i_{t-1}-0,201 e_{t-1}+0,631 \pi_{t-1}+0,013 y_{t-1}
\end{array}\right.
$$

Assim, ao longo do período em questão, o modelo econômico resume-se, depois das devidas estimativas, ao sistema (7) o qual será estudado à partir da metodologia de sistemas de equações em diferenças.

\footnotetext{
${ }^{3}$ Para maiores detalhes sobre Filtro de Hodrick-Prescott ver [2].

${ }^{4}$ Maiores detalhes sobre a metodologia VAR podem ser encontrados em [2].

${ }^{5}$ Disponível em http://gretl.sourceforge.net/. Acessado em 20/12/2013.
} 


\section{Estabilidade do sistema de equações em diferenças.}

Considerando como na equação (5) as matrizes $A$ e $G$, teremos para o sistema (7) os seguintes autovalores calculados pelo software livre Wolfram alpha ${ }^{6}$.

$$
\begin{aligned}
& \lambda_{1} \approx 0,328+0,301 i \\
& \lambda_{2} \approx 0,328-0,301 i \\
& \lambda_{3} \approx-0,631 \\
& \lambda_{4} \approx-0,142
\end{aligned}
$$

Como os autovalores, individualmente, têm módulo menor que um e a matriz $(I-A)$ é não singular temos, da Expansão de Neumann, que o sistema (7) é assintoticamente estável e converge para o equilíbrio:

$$
(I-A)^{-1} \cdot G=\left[\begin{array}{c}
0,124 \\
-0,066 \\
0,008 \\
0,1
\end{array}\right]
$$

\section{Conclusões}

Os resultados encontrados neste estudo sugerem a existência de equilíbrio para o sistema modelado com dados no período 1999-2013. De acordo com (8), em equilíbrio, o diferencial de juros é negativo, o valor de $y_{t}$ positivo, sugerindo que o produto esteja acima de sua tendência de longo prazo. Em relação a variação de $e_{t}$, os resultados sugerem um aumento da taxa de câmbio (desvalorização) relativamente pequena, e taxa de inflação positiva. O comportamento de $y_{t}$ é compatível com os resultados obtidos para a inflação.

No período considerado, com algumas exceções, as taxas de juros mantiveram tendência de queda. De qualquer forma, o diferencial de juros negativo não é um resultado esperado, considerando-se que historicamente as taxas de juros no Brasil mantêm-se em níveis elevados para os padrões internacionais. De qualquer forma, estes resultados podem ser considerados compatíveis com outras pesquisas sobre o tema utilizando-se de outras metodologias.

A partir destes resultados podem ser realizadas simulações para este sistema, considerando possíveis cenários distintos de condução de políticas econômicas por parte do governo.

\section{Referências}

[1] S. Elaydi, "An introduction to difference equations". 3 rd ed. Springer, 2005.

[2] W. Enders, “Applied econometric time series”. New York: John Wiley \& Sons, 2004.

\footnotetext{
${ }^{6}$ Disponível em http://www.wolframalpha.com/
} 
[3] Fraga et al.. Inflation targeting in emerging market economies. Working Paper - Banco Central do Brasil, $\mathrm{n}^{\circ}$ 76, 2003.

[4] G.H. Franco, B. "O Plano Real e outros ensaios”. Rio de Janeiro: Francisco Alves, 1995.

[5] G. Gandolfo, "Economic Dynamics". Study Edition. Springer, 1997.

[6] Lima, G. T., Setterfield, M. Inflation Targeting and Macroeconomic Stability in a Post Keynesian Economy. Journal of a Post Keynesian Economics, Vol. 30 No. 3, pp. 435-461, Springer, (2008).

[7] Lobo, B. S., Oreiro, J. L. Metas de inflação, Crescimento e EstabilidadeMacroeconômica: Uma análise a partir de um modelo Pós-keynesiano macrodinâmico não-linear. Anais do XXXIX Encontro Nacional de Economia (ANPEC), 2011.

[8] Minella, A. et al.. Inflation targeting in Brazil: Constructing credibility under exchange rate volatility. Working Paper - Banco Central do Brasil, n 77, (2003).

[9] A. Modenesi, M. "Regimes monetários: teoria e experiência do Real". Barueri: Manole, 2005.

[10] Modenesi, A. M., Araújo, E. C. Custos e benefícios do controle inflacionário no Brasil (20002008): uma análise empírica do mecanismo de transmissão da política monetária com base em modelo VAR. Anais do XXXVIII Encontro Nacional de Economia (ANPEC), 2010.

[11] Santos, A. L. M. Inflation targeting in a Post Keynesian economy. Journal of Post Keynesian Economics, Vol.4, No. 2 pp. 295-318, Winter, 2011-12. 